\title{
Precise Velocity Observation of K-giants: Evidence for Solar-Like Oscillations in Arcturus
}

\author{
W. J. Merline \\ Instrumentation and Space Research Division, Southwest Research \\ Institute, Boulder, CO 80302
}

\begin{abstract}
.
High accuracy measurements of variations in the radial velocity of the $\mathrm{K} 1$ giant star Arcturus have been obtained. The observations span 5 years and have a point-to-point repeatability of $5 \mathrm{~m} \mathrm{~s}^{-1}$ and night-tonight stability of better than $20 \mathrm{~m} \mathrm{~s}^{-1}$. Velocity oscillations of Arcturus were discovered during the course of this work in 1986 . Subsequent, extensive additional data, indicate that Arcturus is exhibiting global nonradial acoustic oscillations with characteristics similar to those occurring in the Sun.
\end{abstract}

All observations were done using a radial velocity spectrometer, designed to search for extrasolar planets, at a dedicated facility of the University of Arizona on Kitt Peak. A dedicated facility was crucial to this work - because of the changing nature of the oscillations, many observing runs, over several years, were required to understand the star's behavior. Continuous data sets as long as 30 days were acquired. Preliminary pulsation models were performed in collaboration of Art Cox at Los Alamos National Lab.

The velocity power spectra are complicated and variable. There is substantial evidence that the variations are solar-like $p$-mode oscillations. At least 10 frequencies have been identified, over the range 8.3 to 1.7 days. A spectrum of evenly spaced modes is apparent, yielding a value for $\Delta \nu_{0} \approx 1.2 \mu \mathrm{Hz}$. The average power spectrum peaks near 3 days. There is a broad envelope of power with a distribution reminiscent of that seen in the Sun. Both the mode spacing and the period of peak power are consistent with scaling from the Sun (Kjeldsen \& Bedding 1995). The oscillations appear to undergo abrupt discontinuities and have phase coherence times of a few weeks. We interpret the driving to be due to stochastic excitation by convection.

Recent observations of the G5 IV star $\eta$ Boo by Brown et al. (1997) have failed to confirm the detection of $p$-mode oscillations reported by Kjeldsen et al. (1995) Thus, Arcturus may be one of the first stars known to exhibit solar-like oscillations. If other K-giant variables can be shown to exhibit similar oscillations, Arcturus may represent the prototype for a new class of variable stars. We know some other K-giants are variable on short time scales (Hatzes and Cochran 1994b; Edmonds \& Gilliland 1996), but many are not (Horner 1996). 


\section{Introduction}

Definitive evidence for solar-like oscillations in other stars has eluded observers for nearly two decades. The work of Traub, Mariska, \& Carleton (1978) failed to show conclusive evidence of oscillations. Tentative detections of other oscillations have been reported in $\epsilon$ Eri (K2 V) (Noyes et al. 1984), and $\alpha$ Cen A (G2 V) and $\alpha$ CMi (F5 IV) (Gelly, Grec, \& Fossat 1986; Brown \& Gilliland 1990; Brown et al. 1991). Kjeldsen et al. (1995), using a novel method of monitoring changes in the equivalent widths of temperature-sensitive lines, have reported $p$-mode oscillations in the G0 IV star $\eta$ Boo, which they term "the first clear evidence of solar-like oscillations in a star other than the Sun". However, recent observations of $\eta$ Boo by Brown et al. (1997) have failed to confirm this detection.

With the recent exciting results from GONG and SOHO, and with the instrumentation currently able to study oscillations, at least in K-giants (and possibly just now getting to the edge of suitability for solar-type stars), observers and theoreticians are becoming more anxious for evidence of other solar-like oscillations. While non-radial oscillations also appear in other stars, such as white dwarfs, $\delta$ Scuti, and rapidly oscillating Ap stars, they are thought to be driven by different mechanisms than in the Sun.

Seismic inferences about the solar interior structure and processes gained by the study of solar oscillation data have been astounding. Using these data, we can probe the Sun, learning primarily about the sound speed with depth, but consequently about the depth of the convection zone, mechanical mixing activities deep within the Sun, and solar rotation with depth and hence about the solar dynamo and activity cycles. The richness of information now being gleaned from solar oscillations, more than 30 years after their discovery, and more than 20 years after they became understood, is yielding new insights into the structure of the Sun that is not available by other means. While we cannot hope to learn as much from other stars, because they are fainter and have unresolved disks, we can use them as probes of the effects of stellar evolution.

While one is initially drawn to solar-type stars, one must at the same time realize the difficulty of those studies - exceedingly low amplitudes $\left(\sim 1 \mathrm{~m} \mathrm{~s}^{-1}\right)$ in relatively faint stars. While observations of these stars can and must be done, one should also look to those closely-related stars, K-giants for example, evolved stars of approximately one solar mass, which may also show similar oscillations. The expected amplitudes of oscillations $\left(\sim 60 \mathrm{~m} \mathrm{~s}^{-1}\right.$; Kjeldsen \& Bedding 1995) in these stars are well within the capabilities of present instrumentation. The focus in this paper is on short period oscillations (a few days) as opposed to the longer-period variations (periodic or semi-periodic) variations that are now becoming well known in K-giants. This class of variation is discussed, in this volume, by Larson, Yang, \& Walker and by Cummings \& Hearnshaw. Furthermore, the possibility that non-radial oscillations might mimic the effect of a planetary companion, as was suggested (but now disproven) by Gray (1997) for $51 \mathrm{Peg}$, means that to understand planetary signatures, we must understand solar-like oscillations in other stars.

In the H-R diagram, K-giant stars are surrounded by many different classes of variable stars. In that sense, it may be surprising that these stars had not been observed to be variable. But besides the historical lack of observational evidence for variability, there were also theoretical reasons to believe that these stars 
should be stable. Stars redward of the Cepheid instability strip were thought to have stability returned because of the onset of efficient convection, interfering with the deep envelope-ionization mechanism responsible for driving the Cepheid oscillations. In addition, the K-giants were too blue to begin to pick up instability from the higher-level H-ionization, which is thought to drive the red variables, despite deep convection.

\section{Observational Technique}

Our instrument is an interferometrically-calibrated spectrometer, maintained in a temperature-regulated clean-room. The telescope is a dedicated $0.9 \mathrm{~m}$ Newtonian, located on Kitt Peak. Starlight is delivered to the spectrometer by means of an optical fiber from the telescope focal plane. The interferometer is a tilttunable Fabry-Perot, with spectral dispersion accomplished by a cross-dispersed échelle. We sample about 750 points in the stellar spectrum over the wavelength range 4250-4750 $\AA$, using 19 échelle orders. The Fabry-Perot transmission function modulates the spectrum, resulting in a series of well-separated spots (images of an approximately circular entrance aperture) on the CCD detector. Each of these spots then has a well-defined wavelength, fixed by the Fabry-Perot. As the star moves with respect to the telescope, the spectrum shifts with respect to this fixed-wavelength grid. For points that fall on the steep slopes of spectral lines, the intensity will be sen to shift up or down, depending on the sign of the slope of the spectrum at that point. If one knows this slope (which we measure by scanning the Fabry-Perot at an earlier time), then the process of measuring the stellar velocity is reduced to one of doing photometry on the many "spots" on the detector. The stability of the Fabry-Perot is monitored by frequent calibration with an $\mathrm{Fe}-\mathrm{Ar}$ hollow-cathode emission source and by regular observation of a lunar crater. The system was able to routinely achieve random errors of about $5 \mathrm{~m} \mathrm{~s}^{-1}$ on Arcturus. The blue portion of the spectrum is chosen specifically to maximize the information content for measuring radial velocities (Merline 1985). Although this instrument was designed to search for planetary systems around solar-type stars, it has always worked better on K-giants, because of the high density of sharp spectral lines (uncontaminated by telluric lines) in the blue portion of the spectrum. The instrument and technique are described in McMillan et al. (1986).

\section{Discussion of Arcturus}

In 1986, we reported our discovery of oscillations in Arcturus on times scales of days (Smith, McMillan, \& Merline 1987). We found periodic variations with an amplitude of about $160 \mathrm{~m} \mathrm{~s}^{-1}$ and a period of about 2 days. This initial report was met with substantial skepticism, for the reasons outlined above. But with the advent of instruments such as ours, the limits of detectability had improved such that variations of order $10 \mathrm{~m} \mathrm{~s}^{-1}$ were now attainable, opening a window on new, exciting phenomena that may help us tie together some of the common threads among variable stars and provide us with other examples of solar-like oscillations. 
Since this initial discovery, three groups have confirmed the short-period variability of Arcturus (Cochran 1988, Irwin et al. 1989, Belmonte et al. 1990, and Hatzes \& Cochran 1994a). We continued to observe Arcturus, but with each new observing run, we were seeing different dominant frequencies in the spectrum, but with high confidence that the variations were periodic and significant. The presence of periods other than our original one, of 1.8 days, was noted by the other observers. Radical differences between power spectra of different observing runs was evident - some had power predominantly near 5 days, while others has most power near 2-3 days. We have shown, through our intensive observations of Arcturus, that the power spectra are complicated and variable (Merline 1995).

Earlier measurements by M. Smith $(1982,1983)$, which did not show significant night-to-night changes in velocity, are now more understandable than was once thought, because with observations on only a few nights, one could easily have observed at times when the velocities appeared stable.

Power spectra of combined strings of observing runs, covering many months, did not seem to improve the situation, apparently because of lack of long coherence times for the oscillations. Instead, if one combines the power spectra from individual observing runs into an "average periodogram", then a broad envelope of power is apparent, with a distribution reminiscent of that seen for the solar 5-minute oscillations. The span of frequencies in the envelope is about what one would expect if the oscillations were being driven by stochastic excitation (A. Cox 1991, private communication). If that is the driving mechanism, then the period of average power is indicative of the physical conditions near the top of the atmosphere. Scaling from the Sun (see Kjeldsen \& Bedding 1995), one can show that a peak near 3 days may be expected from the acoustic cut-off frequency. In addition, the oscillations show evidence of abrupt disruption. Localized impulsive acoustic events have been observed on the Sun by Restaino, Stebbins, \& Goode (1993), which they interpret as being related to excitation of the solar 5-minute oscillations. Results of computer simulations of excitation of solar $p$-modes by turbulent convection by Bogdan, Cattaneo, \& Malagoli (1993) have evidence for "stochastic interaction between the modes and the underlying turbulence which causes abrupt, episodic events during which the phase coherence of the modes is lost". They also show model plots of the discontinuities that appear qualitatively similar to some of the abrupt changes seen in our Arcturus data. While one cannot yet rule out discontinuous patterns caused by beating of many modes, there are tantalizing similarities with some of the observed behavior of solar oscillations.

Furthermore, a spectrum of modes, approximately evenly-spaced in frequency, has emerged from the many observing runs on Arcturus. All of the significant frequencies observed by other workers have been observed by our group also, plus more. The spacing yields value for $\Delta \nu_{0}=1.2 \mu \mathrm{Hz}$, approximately as expected by rough scaling from the Sun, again in good agreement with the calculations of Kjeldsen \& Bedding (1995). We have considered and rejected other possible explanations for the variability, such as planetary companions, rotation, large convective cells, or radial-only oscillation.

One can readily estimate the acoustic cut-off frequency to be expected for Arcturus, which is roughly $\omega_{a c}=7.5 \mu \mathrm{Hz}$ (or 1.6 day). Brown and Gilliland 
(1994) indicate that the peak of the envelope power ought to occur at about $\omega_{\max }=0.6 \omega_{a c}=4.4 \mu \mathrm{Hz}$ (or $2.6 \mathrm{day}$ ). The observations here show a peak at about 0.3 day, with little power at periods shorter than 1.7 day, consistent with these estimates.

I will note that the calculations of Ando (1976), often quoted to demonstrate that the "expected" values of the acoustic cut-off are of order an hour, were not, in fact, performed for a star like Arcturus, but instead for stars having higher density, notwithstanding their similar locations in the H-R diagram. A more appropriate reference is to Kjeldsen \& Bedding (1995). Christensen-Dalsgaard \& Frandsen (1983) also provide some theoretical work evaluating the possibility of stochastically-driven oscillations in giant stars.

Some intriguing questions about the solar $p$-mode oscillations were first put forth by Isaak (1976): if the solar $p$-modes are stable (i.e., not driven), then how is it that we can see them? If they are driven, why do their amplitudes remain so small? One may equally well ask similar questions about the oscillations seen on Arcturus. The radial oscillations, at least the first several modes, according to models we have performed with Art Cox, should be driven. They may be present but, in any case, they are not sustained and their amplitudes certainly are not large. One would expect the nonlinear effects that limit the oscillation amplitudes to engage at much higher amplitudes as in other, well known, variable stars - at many tens of $\mathrm{km} / \mathrm{s}$, not at the level of a few tens of $\mathrm{m} / \mathrm{s}$ that is observed on Arcturus. What nonlinear effects operate at such small amplitudes? The solution, as in the case of the solar oscillations, and well-described by Brown (1987), may be found in the effects of the turbulence of convection. It has been suspected for some time that the stabilizing mechanism for stars redward of the Cepheid instability strip is the interference of convection (Cox 1980). The interference comes about by means of the damping caused by turbulent viscosity (Goldreich \& Keeley 1977; Goldreich \& Kumar 1990). But at the same time, some of the energy in the convective motions can be transferred to energy in acoustic oscillations. The amplitudes of the modes are governed by the relative strengths of these two opposing processes.

Acknowledgments. This work was done at the University of Arizona, Department of Planetary Sciences and was supported by grants from NASA and NSF to Robert S. McMillan. We are grateful to Art Cox for guidance in stellar pulsation modeling, and to Steve Becker for producing evolution models.

\section{References}

Ando, H. 1976, Pub. Astron. Soc. Japan, 28, 517

Belmonte, J. A., Jones, A. R., Pallé, P. L., \& Cortés, T. D. 1990, ApJ, 358, 595

Bogdan, T. J., Cattaneo, F., \& Malagoli, A. 1993, ApJ, 407, 316

Brown, T. M. 1987, in Stellar Pulsation: Lecture Notes in Physics 274, eds. A. N. Cox, W. M. Sparks, \& S. G. Starrfield (Berlin: Springer-Verlag), 298

Brown, T. M., \& Gilliland, R. L. 1990, ApJ, 350, 839

Brown, T. M., Gilliland, R. L., Noyes, R. W., \& Ramsey, L. W. 1991, ApJ, 368, 32

Brown, T. M., \& Gilliland, R. L. 1994, Ann. Rev. Astron. \& Astrophys., 32, 37 
Brown, T. M., Kennelly, E. J., Korzennik, S. G., Nisenson, P., Noyes, R. W., \& Horner, S.D. 1997, ApJ, 475, 322

Christensen-Dalsgaard, J., \& Frandsen, S. 1983, Solar Physics, 82, 469

Cochran, W. D. 1988, ApJ, 334, 349

Cox, J. P. 1980, Theory of Stellar Pulsation (Princeton: Princeton University Press)

Edmonds, P. D., \& Gilliland, R. L. 1996, ApJ, 464, L157

Gelly, B., Grec, G., \& Fossat, E. 1986, A\&A, 164, 383

Goldreich, P., \& Keeley, D. A. 1977, ApJ, 212, 243

Goldreich, P., \& Kumar, P. 1990, ApJ, 363, 694

Gray, D. F. 1997, Nature, 385, 795

Hatzes, A. P., \& Cochran, W. D. 1994a, ApJ, 422, 366

Hatzes, A. P., \& Cochran, W. D. 1994b, ApJ, 432, 763

Horner, S. 1996, ApJ, 460, 449

Irwin, A. W., Campbell, B., Morbey, C. L., Walker, G. A. H., \& Yang, S. 1989, PASP, 101, 147

Isaak, G. 1976, Observatory 96, 221

Kjeldsen, H., Bedding, T. R., Viskum, M., \& Frandsen, S. 1995, AJ, 109, 1313

Kjeldsen, H., \& Bedding, T. R. 1995, A\&A, 293, 87

McMillan, R. S., Smith, P. H., Frecker, J. E., Merline, W. J., \& Perry, M. L. 1986, in Instrumentation in Astronomy VI: Proc. SPIE, Vol. 627, ed. D. L. Crawford, 2

Merline, W. J. 1985, in Stellar Radial Velocities: Proc. IAU Colloquium 88, eds. A. G. D. Philip \& D. W. Latham (Schenectady: L. Davis Press), 87

Merline, W. J. 1995, Ph.D. dissertation, University of Arizona, Tucson

Noyes, R. W., Baliunas, S. L., Belserene, E., Duncan, D. K., Horne, J., \& Widrow, L. 1984, ApJ, 285, L23

Restaino, S. R., Stebbins, R. T., \& Goode, P. R. 1993, ApJ, 408, L57

Smith, M. A. 1982, ApJ, 253, 727

Smith, M. A. 1983, ApJ, 265, 325

Smith, P. H., McMillan, R. S., \& Merline, W. J. 1987, ApJ, 317, L79

Traub, W. A., Mariska, J. T., \& Carleton, N. P. 1978, ApJ, 223, 583 